Pacific Journal of Mathematics

CONNECTOR THEORY

(t) 


\section{CONNECTOR THEORY}

\section{Hidegoro NaKano AND KaZUMI NaKano}

Connector theory is a generalization of topology and uniformity. Each reflexive binary relation $U$ of a space $S$ induces a mapping from $S$ to $2^{s}$ wherein $x \in S \rightarrow x U=$ $\{y:(x, y) \in U\} \in 2^{s}$. This mapping is called a connector. A uniformity on $S$ is a set of connectors which meets certain conditions. The results in this paper include a necessary-sufficient condition for a connector-set to induce a unique topology, generalizations of continuous mappings and uniformly continuous mappings and characterizations of the connector-sets which correspond to a specific type of topology, for instance, a compact topology, a pseudo-compact topology.

If $\mathfrak{A}$ is a connector-set on $S$, let $\mathfrak{U}^{*}$ denote the connector-set, $\{U$ : for each $x \in S$, there is $V(x) \in \mathfrak{U}$ such that $x U=x V(x)\}$. The following types of connector-sets are defined.

Cone: $\quad U \leqq V$ and $U \in \mathfrak{A} \Rightarrow V \in \mathfrak{A}$.

Net : $\quad U, V \in \mathfrak{A} \Rightarrow W \leqq U \cap V$ for some $W \in \mathfrak{A}$.

Filter: Cone and net.

Sharp: For each $U \in \mathfrak{A}^{\#}$, there is $V \in \mathfrak{A}$ such that $V \leqq U$.

Prenet: $\mathfrak{Q}^{*}$ is a net.

Topological: For each $U \in \mathfrak{A}$ and for each $x \in S$, there is $V \in \mathfrak{A}$ such that $x V^{2} \subseteq x U$.

Topology: Topological sharp filter.

Pretopology: $\mathfrak{U}^{*}$ is a topological net.

Uniform: For each $U \in \mathfrak{A}$, there is $V \in \mathfrak{U}$ such that $V V^{-1} \leqq U$.

Uniformity: Uniform filter.

Totally bounded: For each $U \in \mathfrak{A}$, there are $x_{i} \in S, \quad i=$ $1,2,3, \cdots, m$ such that $S \subseteq \cup x_{i} U$. 
Bounded: For each $U \in \mathfrak{A}$, there are $x_{i} \in S, i=1,2,3, \cdots, m$ and a positive integer $n$ such that $S \subseteq \cup x_{i} U^{n}$.

Absolutely bounded: For each $U \in \mathfrak{A}$ and for each $X \subseteq S$, there are $x_{i} \in X(i=1,2,3, \cdots, m)$, and a positive integer $n$ such that $X \subseteq$ $\cup x_{i} U^{n}$.

A prenet $\mathfrak{A}$ induces a topology $\mathscr{T}(\mathfrak{U})$ (in the usual sense) which will be called an open-topology. The interior theorem states that $\mathfrak{H}$ is a pretopology if and only if $x$ is an interior point of $x U$ for every $U \in \mathfrak{A}$. A topology corresponds uniquely to an open-topology and vice versa (The topology theorem). A topology $\mathfrak{I}$ induces a compact open-topology if and only if $\mathfrak{I}$ is totally bounded. The compact topology theorem states that compactness of $\mathscr{T}(\mathfrak{U})$ for a uniformity $\mathfrak{U}$ implies that $\mathfrak{U}$ is the strongest uniformity included in $\mathfrak{U}^{\#}$.

Suppose $\mathfrak{A}$ is a connector-set on $S$ and $\mathfrak{D}$ is a connector set on $R$. Let $x M \in R$ denote the image of $x \in S$ by a mapping $M$. Each $U \in \mathfrak{D}$ induces the connector $M U M^{-1}$ on $S$ by $x \rightarrow\{z:(x M, z M) \in$ $U\} . \quad M$ is $\mathfrak{A}$-continuous if every $M U M^{-1}, U \in \mathfrak{D}$ belongs to the sharp filter hull of $\mathfrak{A}$. $M$ is uniformly $\mathfrak{A}$-continuous if they belong to the filter hull of $\mathfrak{A}$. The continuity theorem states that the definition is compatible with the continuity (in the usual sense) of $M$ on the topological space $(S, \mathscr{T}(\mathfrak{A}))$ if $\mathfrak{A}$ is a prenet. The kernel theorem is: $\mathfrak{U}$ is the strongest uniformity included in a topology $\mathfrak{I}$ and $\mathfrak{D}$ is a uniform net, then $\mathfrak{I}$-continuity implies uniform $\mathfrak{U}$-continuity. The strongest uniformity included in a topology $\mathfrak{I}$ is bounded if and only if every $\mathfrak{I}$-continuous real-valued function is bounded (The pseudo-compact theorem).

1. Connector systems. Let $S$ be a space on which we develop generalized structures of topology and uniformity. A mapping $U$ from $S$ to $2^{S}$ is called a connector on $S$ if each $x$ of $S$ belongs to its image $x U$. The inverse $U^{-1}$ of $U$ is a mapping: $x \in$ $S \rightarrow\{y: x \in y U\}$. Let $U$ and $V$ be connectors. We write $U \leqq V$ if $x U \subseteq x V$ for every $x$ of $S$. The connector $U V$ (the product of $U$ and $V)$ is defined by $x \in S \rightarrow U\{y V: y \in x U\}$. $U V$ is denoted by $U^{2}$ if $U=V$. An intersection of connectors $\left\{U_{\lambda}: \lambda \in \Lambda\right\}$ is the connector defined by $x \in S \rightarrow \cap\left\{x U_{\lambda}: \lambda \in \Lambda\right\} \in 2^{S}$ and it is denoted by $\cap U_{\lambda}$. A non-empty set of connectors is called a connector system.

A connector system $\mathfrak{A}$ on $S$ is called a cone if $V \in \mathfrak{N}$ whenever $U \leqq V$ and $U \in \mathfrak{A}$. Let $\mathfrak{H}^{<}=\{U: V \leqq U$ for some $V \in \mathfrak{R}\}$. $\quad \mathfrak{R}^{<}$is a cone, and $\mathfrak{H}^{<} \subseteq \mathfrak{B}$ if $\mathfrak{B}$ is a cone and $\mathfrak{H} \subseteq \mathfrak{B}$.

(1.1) $\quad \mathfrak{U}$ is a cone if and only if $\mathfrak{U}^{<}=\mathfrak{A}$.

(1.2) $\quad \mathfrak{U} \subseteq \mathfrak{U}^{<}$. 

(1.3) $\quad \mathfrak{U}^{\ll}=\mathfrak{U}^{<}\left(\mathfrak{U}^{\ll}=\left(\mathfrak{U}^{<}\right)^{<}\right)$.
(1.4) $\mathfrak{U} \subseteq \mathfrak{B}$ implies $\mathfrak{U}^{<} \subseteq \mathfrak{B}^{<}$.
(1.5) $\left(\cup \mathfrak{A}_{\lambda}\right)^{<}=\cup \mathfrak{A}_{\lambda}^{<}$.

A connector system $\mathfrak{A}$ is called a net if for $U, V \in \mathfrak{A}$, there is $W \in \mathfrak{U}$ such that $W \leqq U \cap V$. Let $\mathfrak{P}^{\times}$denote the set of all finite intersections of connectors of $\mathfrak{A}$. Then $\mathfrak{U}^{\times}$is a net.

(1.6) $\mathfrak{U} \subseteq \mathfrak{A}^{\times}$.

(1.7) $\quad \mathfrak{U}^{\times x}=\mathfrak{A}^{\times}$.

(1.8) $\mathfrak{A} \subseteq \mathfrak{B}$ implies $\mathfrak{A}^{\times} \subseteq \mathfrak{B}^{\times}$.

(1.9) $\left(\cup \mathfrak{A}_{\lambda}\right)^{\times}=\left(\cup \mathfrak{U}_{\lambda}^{\times}\right)^{\times}$.

(1.10) $\mathfrak{A}$ is a net if and only if $\mathfrak{A}^{\times} \subseteq \mathfrak{A}^{<}$.

(1.11) $\quad \mathfrak{H}^{x<}=\mathfrak{A}^{<x}$.

Proof. It is obvious that $\mathfrak{A}^{x<x} \subseteq \mathfrak{A}^{x<}$. Thus, by (1.6), (1.4) and (1.8), we obtain $\mathfrak{H}^{<x} \subseteq\left(\mathfrak{U}^{\times}\right)^{<x} \subseteq \mathfrak{H}^{x<}$. On the other hand, for each $U \in \mathfrak{P}^{\times<}, \quad$ there are $U_{i} \in \mathfrak{A}, \quad i=1,2,3, \cdots, n$ such that $\cap\left\{U_{i}: i=1,2,3, \cdots n\right\} \leqq U$. Let $V_{i}$ denote a connector: $x \in$ $S \rightarrow\left(x U_{i}\right) \cup(x U)$. Then $\quad U_{i} \leqq V_{i}, \quad i=1,2,3, \cdots n$ and $U=$ $\cap\left\{V_{i}: i=1,2,3, \cdots n\right\}$.
(1.12) $\quad A^{x<x<}=\mathfrak{Q}^{x<}$.

Proof. Refer to (1.3), (1.7) and (1.11).

A connector system is called a filter if it is a cone and a net. Every finite intersection of connectors of a filter belongs to the filter. Therefore, a filter $\mathfrak{i}$ is a connector system which satisfies the following conditions.

(1) If $U \in \mathfrak{A}$ and $U \leqq V$ then $V \in \mathfrak{A}$.

(2) If $U, V \in \mathfrak{A}$ then $U \cap V \in \mathfrak{A}$.

(1.13) $\mathfrak{A}$ is a filter if and only if $\mathfrak{A}=\mathfrak{U}^{\times}=\mathfrak{U}^{<}$.

(1.14) $\mathfrak{A}$ is a filter if and only if $\mathfrak{A}=\mathfrak{H}^{\times<}$.

(1.15) $\mathfrak{A}^{<}$is a filter if and only if $\mathfrak{A}$ is a net.

$\mathfrak{H}^{x<}$ is a filter and $\mathfrak{B} \supseteq \mathfrak{A}^{x<}$ if $\mathfrak{B}$ is a filter and $\mathfrak{B} \supseteq \mathfrak{A}$. Therefore, $\mathfrak{A}^{x<}$ is called the filter hull of $\mathfrak{A}$.

(1.16) $\mathfrak{A}^{\times<}=\mathfrak{U}^{<}$if and only if $\mathfrak{A}$ is a net.

A connector system $\mathfrak{A}$ is called sharp if for each connector system, $\{U(x) \in \mathfrak{A}: x \in S\}$, there is $V \in \mathfrak{A}$ such that $x V \subseteq x U(x)$ for every $x \in S$. Let $\mathfrak{A}^{\#}=\{U$ : for each $x \in S$, there is $V(x) \in \mathfrak{A}$ such that $x U=x V(x)\}$. The connector system $\mathfrak{Q}^{*}$ is sharp.

(1.17) $\mathfrak{A}$ is sharp if and only if $\mathfrak{U}^{*} \subseteq \mathfrak{H}^{<}$.

(1.18) $\mathfrak{A}$ is sharp if and only if $\mathfrak{U}^{\#<}=\mathfrak{U}^{<}$. 
Proof. (1.3) and (1.4) $\Rightarrow \mathfrak{U}^{\#} \subseteq \mathfrak{U}^{<}$if and only if $\mathfrak{I}^{\#<}=\mathfrak{U}^{<}$.

(1.19) $\mathfrak{A} \subseteq \mathfrak{U}^{\#}$.

(1.20) $\mathfrak{U}^{\# \#}=\mathfrak{U}^{\#}$.

(1.21) $\mathfrak{U} \subseteq \mathfrak{B}$ implies $\mathfrak{U}^{\#} \subseteq \mathfrak{B}^{\#}$.

(1.22) $\left(\cup \mathfrak{A}_{\lambda}\right)^{\#}=\left(\cup \mathfrak{U}_{\lambda}^{*}\right)^{\#}$.

A connector system is called a prenet if for $U, V \in \mathfrak{A}$ and for each $x \in S$, there is $W \in \mathfrak{U}$ such that $x W \subseteq x U \cap x V$. Every net is a prenet.

(1.23) $\mathfrak{U}^{*}$ is a net if and only if $\mathfrak{A}$ is a prenet.

(1.24) Every sharp prenet is a net.

Proof. (1.10) and (1.23) $\Rightarrow \mathfrak{U}^{\times} \subseteq \mathfrak{U}^{\# \times} \subseteq \mathfrak{U}^{\#<}$ if $\mathfrak{A}$ is a prenet. and (1.10) $\Rightarrow \mathfrak{U}^{\#<} \subseteq \mathfrak{U}^{\ll}=\mathfrak{U}^{<}$if $\mathfrak{U}$ is sharp. Hence, $\mathfrak{A}$ is a net if $\mathfrak{A}$ is a sharp prenet.

(1.25) $\quad \mathfrak{U}^{*<}=\mathfrak{U}^{<\#}$.

Proof. Let $\{V(x): x \in S\}$ be a connector system of $\mathfrak{H}^{<}$. There is $W(x) \in \mathfrak{A}$ for each $V(x)$ such that $W(x) \leqq V(x)$. If $W$ and $U$ are defined respectively, by $W: x \in S \rightarrow x W(x)$ and $U: x \in S \rightarrow x V(x)$, then, $W \leqq U$ and $W \in \mathfrak{U}^{*}$. Hence, $U$ belongs to $\mathfrak{Y}^{\#<}$ if $U \in$ $\mathfrak{Y}^{<\#}$. Conversely let $U$ be a connector of $\mathfrak{Y}^{\#<}$. The connector $U(x)$ is defined, for each $x \in S$, by $U(x): y \in S \rightarrow y U(x)=x U$ if $y=x$ and $y U(x)=S$ otherwise. Then, $x U=x U(x)$ for every $x \in S$, and every $U(x),(x \in S)$, belongs to $\mathfrak{H}^{<}$.

(1.26) $\quad \mathfrak{U}^{* \times} \subseteq \mathfrak{U}^{\times *}$.

Proof. Each $U \in \mathfrak{A}^{\# \times}$ is a finite intersection of connectors $\left\{W_{i}: i=1,2,3, \cdots, n\right\}$ of $\mathfrak{U}^{\#}$. There are $U_{i} \in \mathfrak{A}$ for each $x \in S$, such that $\quad x W_{i}=x U_{i}, \quad i=1,2, \cdots, n . \quad x U=\cap\left\{x W_{i}: i=1,2,3, \cdots, n\right\}=$ $\cap\left\{x U_{i}: i=1,2,3, \cdots, n\right\}=x\left(\cap U_{i}\right)$, hence $U$ belongs to $\mathfrak{A}^{\times \#}$.

(1.27) $\mathfrak{P}^{\# \times \#}=\mathfrak{P}^{\times *}$.

Proof. Refer to (1.19), (1.8), (1.21), (1.26) and (1.20) in order. (1.28) $\mathfrak{P}^{\times \# \times}=\mathfrak{U}^{\times \#}$.

Proof. Refer to (1.6), (1.26) and (1.7).

(1.29) $\mathfrak{P}^{\times \# \times \#}=\mathfrak{A}^{\# \times \# \times}=\mathfrak{U}^{\times \#}$.

Proof. Refer to (1.27) and (1.28).

(1.30) $\quad \mathfrak{A}^{\times \#<}=\mathfrak{U}^{\times \#<\times *<}$.

Proof. $\mathfrak{P}^{\times \#<\times \#<}=\mathfrak{P}^{\times \# \times<\#<}$ by $(1.11)$, and $\mathfrak{P}^{\times \# \times<\#<}=\mathfrak{P}^{\times \# \times \#<}$ by 
(1.25). (1.29) and (1.13) $\Rightarrow \mathfrak{A}^{\times \# \times \# \ll}=\mathfrak{A}^{\times \#<}$. Hence, $\mathfrak{P}^{\times \#<x \#<}=\mathfrak{P}^{\times \#<}$.

(1.31) $\mathfrak{A}$ is a sharp filter if and only if $\mathfrak{A}=\mathfrak{U}^{\times \#<}$.

Proof. An implication of (1.13) and (1.18) is the following. $\mathfrak{A}$ is a sharp filter if and only if $\mathfrak{U}=\mathfrak{U}^{\times}=\mathfrak{U}^{<}=\mathfrak{U}^{*}$. Therefore, $\mathfrak{A}=\mathfrak{U}^{\times *<}$ if $\mathfrak{A}$ is a sharp filter. Conversely, $\mathfrak{U}=\mathfrak{U}^{\times \#<}$ implies $\mathfrak{U}^{\times} \subseteq \mathfrak{U}^{\times \#<}=\mathfrak{U} \subseteq \mathfrak{A}^{\times}$, thus $\mathfrak{U}=\mathfrak{U}^{\times} . \quad \mathfrak{U}=\mathfrak{U}^{*}$ and $\mathfrak{A}=\mathfrak{U}^{<}$can be proved similarly.

$\mathfrak{U}^{\times *<}$ is a sharp filter and $\mathfrak{A}^{\times *<} \subseteq \mathfrak{B}$ if $\mathfrak{B}$ is a sharp filter and $\mathfrak{U} \subseteq \mathfrak{B}$. Therefore, $\mathfrak{U}^{\times *<}$ is called the sharp filter hull of $\mathfrak{A}$.

(1.32) $\mathfrak{U}$ is a prenet if and only if $\mathfrak{A}^{\times *<}=\mathfrak{A}^{*<}$.

Proof. $\mathfrak{A}$ is a prenet if and only if $\mathfrak{A}^{*}$ is a net. $\mathfrak{U}^{*}$ is a net if and only if $\mathfrak{A}^{* \times<}=\mathfrak{U}^{*<}$. The last relation implies $\mathfrak{A}^{* \times *}=\mathfrak{P}^{*<*}=\mathfrak{P}^{* * *}=$ $\mathfrak{U}^{*<}$. On the other hand, $\mathfrak{P}^{\# \times *}=\mathfrak{P}^{\#<}$ implies $\mathfrak{P}^{\#<} \subseteq \mathfrak{Y}^{\# \times<} \subseteq \mathfrak{P}^{\# \times<*}=$ $\mathfrak{U}^{*<}$, thus $\mathfrak{I}^{* \times<}=\mathfrak{U}^{*<} . \quad \mathfrak{I}^{* \times<*}=\mathfrak{A}^{\times *<}$ by $(1.25)$ and (1.27).

(1.33) $\mathfrak{U}$ is a sharp prenet if and only if $\mathfrak{I}^{\times \#<}=\mathfrak{P}^{<}$.

Proof. If $\mathfrak{A}$ is a sharp prenet then, by (1.32) and (1.18), $\mathfrak{I}^{\times \#<}=$ $\mathfrak{A}^{*<}=\mathfrak{A}^{<} . \quad \mathfrak{U}^{<} \subseteq \mathfrak{U}^{*<} \subseteq \mathfrak{U}^{\times *<}$, thus, $\mathfrak{H}^{\times *<}=\mathfrak{H}^{<}$implies $\mathfrak{H}^{<}=\mathfrak{U}^{\#<}$.

(1.34) $\mathfrak{U}^{\times *<}=\mathfrak{U}^{*}$ if $\mathfrak{A}$ is a filter.

Proof. Refer to (1.14), (1.25) and (1.31).

2. Base of filters. A connector system $\mathfrak{A}$ is called stronger than a connector system $\mathfrak{B}$ (or $\mathfrak{B}$ is weaker than $\mathfrak{A}$ ) if $\mathfrak{B} \subseteq \mathfrak{A}^{\times<}$.

(2.1) $\mathfrak{A}$ is a net. $\mathfrak{A}$ is stronger than $\mathfrak{B}$ if and only if $\mathfrak{B} \subseteq \mathfrak{H}^{<}$.

(2.2) $\mathfrak{A}$ is stronger and also weaker than $\mathfrak{B}$ if and only if $\mathfrak{U}^{x<}=\mathfrak{B}^{x<}$.

A connector system $\mathfrak{A}$ is called finer than a connector system $\mathfrak{B}$ if $\mathfrak{B} \subseteq \mathfrak{U}^{\times *<}$. (1.28), (1.19), (1.32) and (1.33) imply (2.3), (2.4), (2.5) and (2.6) respectively.

(2.3) $\mathfrak{A}$ is finer than $\mathfrak{B}$ if and only if $\mathfrak{A}^{\times *}$ is stronger than $\mathfrak{B}$.

(2.4) $\mathfrak{A}$ is finer than $\mathfrak{B}$ if $\mathfrak{A}$ is stronger than $\mathfrak{B}$.

(2.5) $\mathfrak{A}$ is a prenet. $\mathfrak{A}$ is finer than $\mathfrak{B}$ if and only if $\mathfrak{B} \subseteq \mathfrak{A}^{*<}$.

(2.6) If $\mathfrak{A}$ is a sharp prenet and finer than $\mathfrak{B}$ then $\mathfrak{A}$ is stronger than $\mathfrak{B}$.

Let $\mathfrak{F}$ be a filter on $S$. A connector system $\mathfrak{A} \subseteq \mathfrak{F}$ is called a basis of $\mathfrak{F}$ if for each $U \in \mathfrak{F}$, there is $V \in \mathfrak{A}$ such that $V \leqq U$.

(2.7) $\mathfrak{A}$ is a basis of $\mathfrak{F}$ if and only if $\mathfrak{F}=\mathfrak{A}^{<}$.

(2.8) The following two statements are equivalent.

(1) $\mathfrak{A}$ is a basis of $\mathfrak{F}$.

(2) $\mathfrak{A}$ is a net, and $\mathfrak{A}$ is stronger and weaker than $\mathfrak{乛}$. 
Proof. The statement (2) is, by (1.16) and (2.27), equivalent to $\mathfrak{A}^{<}=\mathfrak{A}^{\times<}=\mathfrak{F}^{\times<}$. These relations follow if $\mathfrak{r}=\mathfrak{A}^{<}$and $\mathfrak{r}=\mathfrak{F}^{\times<}$, hence, the statement (1) implies the statement (2). The converse is obvious.

(2.9) $\mathfrak{A}$ is a basis of $\mathfrak{U}^{<}$if $\mathfrak{A}$ is a net.

Proof. Refer to (1.15) and (2.7).

(2.10) Every basis of a sharp filter is sharp.

Proof. If $\mathfrak{A}$ is a basis of a sharp filter $\mathfrak{f}$ then, since (1.31) and (2.7) imply $\mathfrak{F}_{\mathfrak{F}}=\mathfrak{r}^{*}$ and $\mathfrak{U}^{<}=\mathfrak{F}$ respectively, $\mathfrak{P}^{*<}=\mathfrak{U}^{<*}=\mathfrak{F}^{*}=\mathfrak{r}=$ $\mathfrak{H}^{<}$. Hence, by (1.18), $\mathfrak{A}$ is sharp.

A connector system $\mathfrak{A}$ is called a prebasis of a filter $\mathfrak{r}$ if $\mathfrak{Q}^{*}$ is a basis of $\mathfrak{i s}$.

(2.11) $\mathfrak{A}$ is a prebasis of $\mathfrak{F}$ if and only if $\mathfrak{U}^{\#<}=\mathfrak{F}$.

(2.12) A filter $\mathfrak{i s}$ is sharp if there is a prebasis.

(2.13) Every basis of $\mathfrak{f}$ is a prebasis if $\mathfrak{f}$ is a sharp filter.

(2.14) Every prebasis is a prenet.

Proof. Refer to (1.23) and (2.8).

(2.15) $\mathfrak{B}$ is a prebasis of $\mathfrak{A}^{\times \#<}$ if and only if $\mathfrak{B}$ is a prenet and $\mathfrak{A}^{\times *<}=\mathfrak{B}^{\times *<}$.

Proof. An implication of (2.11) is the following. $\quad \mathfrak{B}$ is a prebasis of $\mathfrak{A}^{\times *<}$ if and only if $\mathfrak{A}^{\times *<}=\mathfrak{B}^{*<}$. (1.32) states that $\mathfrak{B}$ is a prenet if and only if $\mathfrak{B}^{\times *<}=\mathfrak{B}^{*<} . \quad \mathfrak{P}^{\times *<}=\mathfrak{B}^{*<} \Rightarrow \mathfrak{U}^{\times *<}=\mathfrak{B}^{*<} \subseteq \mathfrak{B}^{\times *<}$ and $\mathfrak{B}^{\times *<} \subseteq$ $\mathfrak{B}^{*<\times *<}=\mathfrak{I}^{\times *<\times *<}=\mathfrak{I}^{\times *<}$. Therefore, $\mathfrak{A}^{\times *<}=\mathfrak{B}^{*<}$ if and only if $\mathfrak{B}^{\times *<}=$ $\mathfrak{B}^{*<}$ and $\mathfrak{I}^{\times *<}=\mathfrak{B}^{\times *<}$.

(2.16) $\mathfrak{F}$ is a sharp filter. A connector system $\mathfrak{A} \subseteq \mathfrak{F}$ is a prebasis of $\mathfrak{F}$ if and only if $\mathfrak{A}$ is a prenet and finer than $\mathfrak{x}$.

The following two statements are equivalent if $\tilde{s}$ and (S) are filters.

(1) $\mathfrak{i s}$ is stronger than (3).

(2) $\mathfrak{\wp} \supseteq$ (5).

An intersection of filters is a filter. Therefore, for each set of filters $\left\{\mathfrak{F}_{\lambda}: \lambda \in \Lambda\right\}$, there exists the strongest filter among the filters weaker than all $\mathfrak{r}_{\lambda}, \lambda \in \Lambda$. Likewise, there exists the weakest filter among the filters stronger than all $\mathfrak{r}_{\lambda}, \lambda \in \Lambda$. The two filters are respectively denoted by $\wedge \mathfrak{F}_{\lambda}$ and $\vee \widetilde{R}_{\lambda}$.

(2.19) If $\mathfrak{A}_{\lambda}$ is a basis of $\mathfrak{F}_{\lambda}$ for each $\lambda \in \Lambda$ then $\left(\cup \mathfrak{A}_{\lambda}\right)^{\times}$is a basis of $\vee \mathfrak{r}_{\lambda}$.

Proof. The following relations are implied respectively by (1.3), (1.11), (1.5), (2.7) and (2.17). $\left(\cup \mathfrak{A}_{\lambda}\right)^{x<}=\left(\cup \mathfrak{A}_{\lambda}\right)^{x \ll}=\left(\cup \mathfrak{A}_{\lambda}\right)^{<x<}=$ $\left(\cup \mathfrak{U}_{\lambda}^{<}\right)^{x<}=\left(\cup \mathfrak{F}_{\lambda}\right)^{x<}=\vee \mathfrak{F}_{\lambda}$. Hence, by $(2.7),\left(\cup \mathfrak{U}_{\lambda}\right)^{\times}$is a basis of $\vee \mathfrak{F}_{\lambda}$. (2.20) $\left(\vee \widetilde{r}_{\lambda}\right)^{*}=\left(\cup \widetilde{r}_{\lambda}\right)^{x *}$. 
(2.21) if $\mathfrak{A}_{\lambda}$ is a prebasis of $\mathfrak{\aleph}_{\lambda}$ for each $\lambda \in \Lambda$, then $\left(\cup \mathfrak{P}_{\lambda}\right)^{\times}$is a prebasis of $\left(\vee \widetilde{r}_{\lambda}\right)^{*}$.

Proof. $\mathfrak{l}_{\lambda}=\mathfrak{P l}_{\lambda}^{*<}, \lambda \in \Lambda$ and $\left(\vee \mathfrak{r}_{\lambda}\right)^{*}=\left(\cup \mathfrak{r}_{\lambda}\right)^{\mathrm{*}<<}$ by $(2.11)$ and (2.20). (1.22) and (1.27) $\Rightarrow\left(\cup \mathfrak{P}_{\lambda}^{*}\right)^{\times \#}=\left(\cup \mathfrak{P}_{\lambda}\right)^{\times \#}$. Therefore,

$\left(\vee \mathfrak{r}_{\lambda}\right)^{*}=\left(\cup \mathfrak{P}_{\lambda}^{*<}\right)^{\times *<}=\left(\cup \mathfrak{P}_{\lambda}^{*}\right)^{<\times *<}=\left(\cup \mathfrak{Y}_{\lambda}^{*}\right)^{\times \# \ll}=\left(\cup \mathfrak{I}_{\lambda}^{* *}\right)^{\mathrm{x} *<}$ $=\left(U \mathfrak{V}_{\lambda}\right)^{\times \#<}$.

(2.22) $\left(v \mathfrak{T}_{\lambda}\right)^{*}=\left(\vee \mathfrak{T}_{\lambda}^{*}\right)^{*}$.

(2.22) is a generalization of Theorem 5 in $\S 28$ of [1].

3. Topologies. A connector system $\mathfrak{A}$ is called topological if for each $U \in \mathfrak{P}$ and for each $x \in S$, there is $V \in \mathfrak{A}$ such that $x V^{2} \subseteq x U$.

(3.1) If $\mathfrak{A}$ is topological then $\mathfrak{A}^{<}, \mathfrak{A}^{\times}$and $\mathfrak{U}^{*}$ are topological.

(3.2) If $\mathfrak{A}_{\lambda}, \lambda \in \Lambda$ are all topological then $\cup \mathfrak{I}_{\lambda}$ is topological.

A topological sharp filter is called a topology; i.e., a topology $\mathfrak{I}$ on $S$ is a connector system which satisfies the following conditions:

(1) If $U, V \in \mathfrak{I}$ then $U \cap V \in \mathfrak{I}$.

(2) If $U \leqq V$ and $U \in \mathfrak{I}$ then $V \in \mathfrak{T}$.

(3) For each system $\{U(x) \in \mathfrak{I}: x \in S\}$, there exists $U \in \mathfrak{I}$ such that $x U=x U(x)$ for every $x \in S$.

(4) For each $U \in \mathcal{I}$ and for each $x \in S$, there exists $V \in \mathcal{I}$ such that $x V^{2} \subseteq x U$.

The use of the word "topology" is not conventional. Compatibility of the terminology is cleared in the following section. Topologies are filters, hence, a topology is stronger than another if and only if the former includes the latter. A topology hull of a connector system $\mathfrak{A}$ is the weakest topology among the topologies stronger than $\mathfrak{A}$. A topology hull is unique if it exists.

(3.3) $\mathfrak{A}^{\times *<}$ is the topology hull of $\mathfrak{A}$ if $\mathfrak{U}$ is topological.

Proof. Refer to (1.31) and (3.1).

(3.4) If one of $\mathfrak{H}, \mathfrak{U}^{\times}, \mathfrak{H}^{<}$and $\mathfrak{Y}^{*}$ has a topology hull, then all of them have the same topology hull.

Proof. If $\mathfrak{I}$ is a topology and $\mathfrak{I}$ includes $\mathfrak{A}$ then $\mathfrak{I}$ includes all $\mathfrak{H}^{<}$, $\mathfrak{P}^{\times}$and $\mathfrak{H}^{*}$ because $\mathfrak{I}=\mathfrak{T}^{\times *<}$. If $\mathfrak{T}$ includes one of $\mathfrak{T}^{<}, \mathfrak{P}^{\times}$and $\mathfrak{T}^{*}$ then $\mathfrak{I}$ includes $\mathfrak{A}$, thus $\mathfrak{I}$ includes the other two.

(3.5) $\mathfrak{I}^{*}$ is the topology hull of $\mathfrak{P}$ if $\mathfrak{I}$ is a topological filter.

Proof. Refer to (1.34) and (3.3).

(3.6) $\mathfrak{A}^{<}$is the topology hull of $\mathfrak{A}$ if and only if $\mathfrak{Y}$ is topological sharp prenet. 
Proof. Refer to (3.3) and (1.33). $\mathfrak{A}$.

(3.7) If $\mathfrak{U}$ is a topological prenet, then $\mathfrak{U}^{\#<}$ is the topology hull of

Proof. Refer to (3.3) and (1.32).

(3.8) Every basis of a topology $\mathfrak{T}$ is a topological sharp prenet, and $\mathfrak{I}$ is its topology hull.

Proof. $\quad \mathfrak{I}=\mathfrak{P}^{<} \Rightarrow \mathfrak{A}^{\times *<}=\mathfrak{H}^{<\times *<}=\mathfrak{I}^{\times *<}=\mathfrak{T}=\mathfrak{H}^{<}$. A basis of a topology is topological, hence, $\mathfrak{I}$ is the topology hull of $\mathfrak{A}$.

(3.9) A topology $\mathfrak{T}$ is the topology hull of every prebasis of $\mathfrak{T}$.

(3.10) If $\mathfrak{I}_{\lambda}$ is the topology hull of $\mathfrak{H}_{\lambda}$ for each $\lambda \in \Lambda$ then $\left(\vee \mathfrak{T}_{\lambda}\right)^{*}$ is the topology hull of $\cup \mathfrak{P A}_{\lambda}$.

Proof. $\left(\vee \mathfrak{T}_{\lambda}\right)^{*}$ is the topology hull of $\cup \mathfrak{T}_{\lambda}$ by (3.2), (3.3) and (2.20). If $\mathfrak{I}$ is a topology which includes all $\mathfrak{A}_{\lambda}, \lambda \in \Lambda$ then $\mathfrak{I}$ includes all $\mathfrak{I}_{\lambda}, \lambda \in \Lambda$. Hence the both unions have the same topology hull.

A connector system $\mathfrak{A}$ is called a pretopology if $\mathfrak{U}^{*}$ is a topological net. Every topology is, by (3.1), a pretopology.

(3.11) Every pretopology is a prenet.

(3.12) Every topological prenet is a pretopology.

(3.13) If $\mathfrak{Y}$ is a pretopology then $\mathfrak{V}^{\#<}$ is the topology hull of $\mathfrak{A}$ and $\mathfrak{A}$ is a prebasis of $\mathfrak{Q}^{*<}$.

Proof. (1.27) and (3.1) $\Rightarrow \mathfrak{U}^{\times *<}$ is topological if $\mathfrak{A}$ is a pretopology. Then, $\mathfrak{A}^{\times *<}$ is the topology hull of $\mathfrak{A}$. $\mathfrak{U}^{\times *<}=\mathfrak{A}^{*<}$ by (3.11) and (1.32), hence, $\mathfrak{X}^{*<}$ is the topology hull of $\mathfrak{A}$.

(3.14) If every $\mathfrak{A}_{\lambda}, \lambda \in \Lambda$ is a pretopology then $\left(\cup \mathfrak{A}_{\lambda}\right)^{\times}$is a pretopology.

Proof. (1.27) and (1.22) $\Rightarrow\left(\cup \mathfrak{A}_{\lambda}\right)^{\times *}=\left(\cup \mathfrak{A}_{\lambda}^{*}\right)^{\# \times \#}$, and (3.1) and (3.2) $\Rightarrow\left(\cup \mathfrak{P}_{\lambda}^{*}\right)^{* \times \#}$ is topological if $\mathfrak{H}_{\lambda}^{*}, \lambda \in \Lambda$ are topological. $\quad\left(\cup \mathfrak{A}_{\lambda}\right)^{\times \#}$ is a net because $\left(\cup \mathfrak{U}_{\lambda}\right)^{\times *}=\left(\cup \mathfrak{A}_{\lambda}\right)^{\times * x}$. Hence, $\left(\cup \mathfrak{A}_{\lambda}\right)^{\times}$is a pretopology.

4. Open sets. The word 'topology' has been already used for a connector system. To avoid confusion, a topology in usual sense is called an open-topology. An open-topology $\mathscr{T}$ is a family of subsets of $S$ and

(1) $X_{\lambda} \in \mathscr{T}, \lambda \in \Lambda \Rightarrow \cup X_{\lambda} \in \mathscr{T}$,

(2) $X, Y \in \mathscr{T} \Rightarrow X \cap Y \in \mathscr{T}$,

(3) $\varnothing, S \in \mathscr{T}$.

Each prenet $\mathfrak{A}$ on $S$ induces an open-topology in the following way. A set $X$ of $S$ is an open set if for each $x \in X$, there is $U \in \mathfrak{A}$ such that $x U \subseteq X$. This open-topology is denoted by $\mathscr{T}(\mathfrak{A})$. 
(4.1) $\mathfrak{A}$ and $\mathfrak{B}$ are prenets. Then $\mathscr{T}(\mathfrak{B}) \subseteq \mathscr{T}(\mathfrak{U})$ if $\mathfrak{U}$ is finer than $\mathfrak{B}$, and $\mathscr{T}(\mathfrak{U})=\mathscr{T}(\mathfrak{B})$ if $\mathfrak{O}^{*<}=\mathfrak{B}^{*<}$.

Proof. (2.5) states that $\mathfrak{B} \subseteq \mathfrak{A}^{*<}$ if a prenet $\mathfrak{A}$ is finer than $\mathfrak{B}$. Thus, for each $U \in \mathfrak{B}$ and for each $x \in S$, there is $V \in \mathfrak{A}$ such that $x V \subseteq x U$. Hence, $\mathscr{T}(\mathfrak{B}) \subseteq \mathscr{T}(\mathfrak{A})$. If $\mathfrak{U}^{*<}=\mathfrak{B}^{*<}$ then $\mathfrak{A} \subseteq \mathfrak{B}^{*<}$. and $\mathfrak{B} \subseteq$ $\mathfrak{Q}^{*<}$, thus, $\mathscr{T}(\mathfrak{H})=\mathscr{T}(\mathfrak{B})$.

(4.2) $\quad \mathscr{T}(\mathfrak{U})=\mathscr{T}\left(\mathfrak{U}^{<}\right)=\mathscr{T}\left(\mathfrak{U}^{\times}\right)=\mathscr{T}\left(\mathfrak{U}^{*}\right)$.

A connector $U$ is called $\mathfrak{A}$-open if $x U \in \mathscr{T}(\mathfrak{A})$ for every $x \in S$.

(4.3) If a connector is $\mathfrak{A}$-open then the connector belongs to $\mathfrak{H}^{\#<}$.

$\mathfrak{A}$-Int $X$ denotes the interior of a set $X \subseteq S$, relative to the open-topology $\mathscr{T}(\mathfrak{U})$, i.e., $A$-Int $X=\cup\{Y \in \mathscr{T}(\mathfrak{P}): Y \subseteq X\}$. $\mathscr{T}(\mathfrak{B})$.

(4.4) $\mathfrak{A}$-Int $X=\mathfrak{B}$-Int $X$ for every $X \subseteq S$ if and only if $\mathscr{T}(A)=$

INTERIOR THEOREM. The following statements are equivalent.

(1) $\mathfrak{A}$ is a pretopology.

(2) $\mathfrak{U}$-Int $X=\{x ; x U \subseteq X$ for some $U \in \mathfrak{A}\}$ for every subset $X \subseteq S$.

(3) If $U \in \mathfrak{A}$ then $x$ belongs to the $\mathfrak{A}$-interior of $x U$ for every $x \in S$.

Proof. (1) $\Rightarrow$ (2). Let $X \subseteq S$ and let $Y \approx\{x \in S: x V \subseteq X$ for some $\left.V \in \mathfrak{U}^{*}\right\} . \quad x V \subseteq X$ for some $V \in \mathfrak{A}^{*}$ if and only if $x U \subseteq X$ for some $U \in \mathfrak{A}$. Therefore, $Y=\{x \in S: x U \subseteq X$ for some $U \in \mathfrak{A}\}$. We show $Y$ is the $\mathfrak{X}^{*}$-interior of $X$. If $x V \subseteq X$ for some $V \in \mathfrak{A}^{*}$, then, since $\mathfrak{A}^{*}$ is topological, there is $W \in \mathfrak{A}^{*}$ such that $y W \subseteq x V \subseteq X$ for every $y \in x W$. Therefore $x W \subseteq Y$ for some $W \in \mathfrak{Q}^{\#}$ if $x \in Y$. This implies $Y \in \mathscr{T}\left(\mathfrak{Q}^{*}\right)$. If $Z \subseteq X$ and $Z \in \mathscr{T}\left(\mathfrak{P}^{*}\right)$ then $Z \subseteq Y$, hence, $Y$ is the $\mathfrak{U}^{*}$-interior of $X$. $\mathfrak{A}$-Int $X=\mathfrak{A}^{*}$-Int $X$ by (4.2) and (4.4), thus $\mathfrak{X}$-Int $X=\{x \in S: x U \subseteq X$ for some $U \in \mathfrak{A}\}$. (2) obviously implies (3). (3) $\Rightarrow(1)$ : If $\mathfrak{A}$ is a prenet then $\mathfrak{Q}^{*}$ is a net. Therefore, it is sufficient to show that $\mathfrak{A}^{*}$ is topological. If $x \in S$ and $U \in \mathfrak{Y}^{*}$ then, since $x \in \mathfrak{A}$-Int $(x U)$, there exists $Y \in \mathscr{T}(\mathfrak{H})$ such that $x \in Y \subseteq$ $x U$. For each $y \in Y$, there is $U(y) \in \mathfrak{A}$ such that $y U(y) \subseteq Y$. Define $V$ by $y V=y U(y)$ if $y \in Y$ and $y V=y U$ otherwise. Then $V \in \mathfrak{A}^{*}$ and $x V^{2} \subseteq x U$. Hence $\mathfrak{A}^{*}$ is topological.

(4.5) If $\mathscr{T}(\mathfrak{B}) \subseteq \mathscr{T}(\mathfrak{A})$ and $\mathfrak{B}$ is a pretopology then $\mathfrak{P}$ is finer than $\mathfrak{B}$.

Proof. If $U \in \mathfrak{B}$ then, by the interior theorem, the connector $V: x \rightarrow \mathfrak{B}$-Int $(x U)$ is a $\mathfrak{B}$-open connector. $\quad V$ is also $\mathfrak{A}$-open because $\mathscr{T}(\mathfrak{B}) \subseteq \mathscr{T}(\mathfrak{U}) . \quad V \subseteq U$ and $V \in \mathfrak{A}^{*<}$, hence $U \in \mathfrak{A}^{*<}$. 
Basis Theorem. $\mathfrak{I}$ is a topology. Then, the set of all $\mathfrak{I}$-open connectors is a basis of $\mathfrak{I}$.

Proof. A mapping $V: x \in S \rightarrow \mathfrak{I}$-Int $(x U)$ is a connector if $U \in$ I. $V$ is $\mathfrak{I}$-open and $V \leqq U$. (4.3) implies $V \in \mathfrak{T}$. Hence, the set of all $\mathfrak{I}$-open connectors is a basis of $\mathfrak{I}$.

Comparison TheOREM. $\mathfrak{A}$ and $\mathfrak{B}$ are topologies. $\mathfrak{B} \subseteq \mathfrak{A}$ if and only if $\mathscr{T}(\mathfrak{B}) \subseteq \mathscr{T}(\mathfrak{H})$.

Proof. $\mathfrak{A}$ is finer than $\mathfrak{B}$ if and only if $\mathfrak{B} \subseteq \mathfrak{A}$. Hence, (4.1) implies the one direction and (4.5) implies the other.

Topology THEOREM. $\mathscr{T}$ is an open topology on $S$. There is a unique topology $\mathfrak{T}$ such that $\mathscr{T}=\mathscr{T}(\mathfrak{T})$.

Proof. Let $\mathfrak{A}=\{U ; x U \in \mathscr{T}$ for every $x \in S\} . \quad U$ is a connector and $x \in S$. Define $V$ by $y V=x U$ if $y \in x U$ and $y V=S$ otherwise. Then, $x V^{2}=x U$ and $V$ belongs to $\mathfrak{A}$ if $U$ does. Therefore, $\mathfrak{A}$ is topological. $\mathfrak{A}$ is a sharp net because $\mathfrak{A}=\mathfrak{A}^{\times}=$ $\mathfrak{U}^{*}$. Let $\mathfrak{I}=\mathfrak{A}^{<}$. Then $\mathscr{T}(\mathfrak{T})=\mathscr{T}(\mathfrak{U})$ and $\mathfrak{I}$ is, by (3.6), the topology hull of $\mathfrak{A} . \quad \mathscr{T}=\mathscr{T}(\mathfrak{U})$ is clear by the definition of $\mathfrak{A}$, hence, $\mathscr{T}=$ $\mathscr{T}(\mathfrak{I})$. The comparison theorem implies the uniqueness.

5. Uniformities. A connector system $\mathfrak{A}$ is called uniform if for each $U \in \mathfrak{A}$, there is $V \in \mathfrak{A}$ such that $V V^{-1} \leqq U$. If $\mathfrak{A}$ is uniform, then for each $U \in \mathfrak{A}$, there are $V, W \in \mathfrak{A}$ such that $W W^{-1} \leqq V$ and $V V^{-1} \leqq U . \quad W^{-1} \leqq V$ implies $W \leqq V^{-1}$, thus $W^{2} \leqq V V^{-1} \leqq U$.

(5.1) Every uniform system is topological.

(5.2) If $\mathfrak{A}$ is uniform, then $\mathfrak{H}^{<}$and $\mathfrak{H}^{\times}$are uniform.

(5.3) If $\mathfrak{A}_{\lambda}, \lambda \in \Lambda$ are uniform then $\cup \mathfrak{H}_{\lambda}$ is uniform.

A uniformity is a filter which is uniform. ([1], [2]). If $\mathcal{U}$ is the weakest among the uniformities stronger than $\mathfrak{A}$, then $\mathbb{U}$ is called the uniformity hull of $\mathfrak{A}$.

(5.4) The filter hull $\mathfrak{A}^{x<}$ is the uniformity hull of $\mathfrak{A}$ if $\mathfrak{A}$ is uniform.

(5.5) $\mathbb{U}$ is a uniformity. Then, a basis $\mathfrak{B}$ of $\mathfrak{U}$ is a uniform net and $\mathbb{U}$ is the uniformity hull of $\mathfrak{B}$.

(5.6) If $\mathfrak{A}$ is a uniform net then $\mathfrak{A}^{<}$is the uniformity hull and $\mathfrak{A}$ is a basis of $\mathfrak{U}^{<}$.

Proof. Refer to (1.16), (5.4) and (2.7).

(5.7) If $\mathfrak{U}_{\lambda}$ is the uniformity hull of $\mathfrak{A}_{\lambda}, \lambda \in \Lambda$, then $\vee \mathfrak{U}_{\lambda}$ is the uniformity-hull of $\cup \mathfrak{A}_{\lambda}$. 
Proof. (5.3) and (5.4) $\Rightarrow \vee \mathfrak{U}_{\lambda}$ is a uniformity. If $\mathbb{U}$ is a uniformity and $\mathbb{U}$ includes all $\mathfrak{I}_{\lambda}, \lambda \in \Lambda$ then $\mathfrak{U}$ includes all $\mathfrak{U}_{\lambda}, \lambda \in \Lambda$ and $\mathfrak{U}=\mathfrak{H}^{x<}$. Hence, $\mathfrak{U}$ includes $\left(\cup \mathfrak{U}_{\lambda}\right)^{x<}=\vee \mathfrak{U}_{\lambda}$.

$\mathfrak{A}$ is a connector system. If there is a uniformity which is the strongest among the uniformities weaker than $\mathfrak{A}$ then the uniformity is called the uniformity kernel of $\mathfrak{A}$. A uniformity kernel is unique if it exists.

(5.8) Every filter has a uniformity kernel.

Proof. Every filter includes the uniformity which contains the only connector, $x \in S \rightarrow S$ (for every $x \in S$ ). Let $\{\mathfrak{U}: \mathfrak{U} \subseteq \mathfrak{F}\}$ denote the collection of uniformities weaker than a filter $\mathfrak{s} . \quad(5.7) \Rightarrow$ $\vee\{\mathfrak{U}: \mathfrak{U} \subseteq \mathfrak{F}\} \quad$ is a uniformity. (2.17) states $\quad \vee\{\mathfrak{U}: \mathfrak{U} \subseteq \mathfrak{F}\}=$ $(\cup\{\mathfrak{H}: \mathbb{U} \subseteq \mathfrak{F}\})^{x<} \subseteq \mathfrak{F}^{x<}=\mathfrak{F}$. Therefore, the uniformity is included in $\mathfrak{s}$ and the strongest among $\{\mathfrak{U}: \mathbb{U} \subseteq \mathfrak{T}\}$.

The topology hull of a uniformity $\mathfrak{U}$ is $\mathbb{U}^{*}$ because $\mathfrak{U}$ is a topological filter. $\mathscr{T}\left(\mathfrak{U}^{*}\right)$ is a uniform topology (called the induced topology in [1] and [2]). Therefore, a topology $\mathfrak{T}$ corresponds to a uniform topology if and only if there is a uniformity $\mathfrak{U}$ such that $\mathfrak{I}=\mathfrak{U}^{*}$.

(5.9) $\mathfrak{I}$ is a topology. There is a uniformity $\mathbb{U}$ such that $\mathfrak{T}=\mathfrak{U}^{*}$ if and only if $\mathfrak{I}$ is the topology hull of its uniformity kernel.

Proof. Since a topology is a filter, a topology $\mathfrak{I}$ has the uniform kernel $\mathfrak{B}$. If $\mathfrak{I}=\mathbb{U}^{*}$ for some uniformity $\mathbb{U}$ then $\mathbb{U} \subseteq \mathfrak{B} \subseteq \mathfrak{T}$. Hence $\mathfrak{I}=\mathfrak{B}^{*}$.

(5.10) $\mathfrak{I}_{\lambda}, \lambda \in \Lambda$ are topologies and $\mathfrak{H}_{\lambda}, \lambda \in \Lambda$ are uniformities. If $\mathfrak{I}_{\lambda}=\mathbb{1}_{\lambda}^{*}$ for each $\lambda \in \Lambda$, then there is a uniformity $\|$ such that $\left(\vee \mathfrak{I}_{\lambda}\right)^{*}=\mathbb{1 1}^{*}$.

Proof. $\left(\vee \mathbb{l}_{\lambda}\right)^{*}=\left(\vee \mathbb{l}_{\lambda}^{*}\right)^{*}=\left(\vee \mathfrak{I}_{\lambda}\right)^{*}$ by (2.22). $\vee \vee \mathbb{l}_{\lambda}$ is a uniformity by (5.7).

6. Mappings. Let $R$ be a space and let $U$ be a connector on $R$. $\quad M$ is a mapping from a space $S$ to $R . \quad x M$ and $(x M) U$ denote the image of $x$ by $M$ and the image of $x M \in R$ by $U$ respectively. Define a connector on $S$ by corresponding $x$ of $S$ to the set $\{y \in S: y M \in$ $(x M) U\}$. This connector is denoted by $M U M^{-1} . x\left(M U M^{-1}\right)$ is the image of $x$ by $M U M^{-1}$. The following formulas are found in [2].

(6.1) $M(U \cap V) M^{-1}=M U M^{-1} \cap M V M^{-1}$.

(6.2) $U \leqq V$ implies $M U M^{-1} \leqq M V M^{-1}$.

(6.3) $\left(M U M^{-1}\right)^{-1}=M\left(U^{-1}\right) M^{-1}$.

(6.4) $\left(M U M^{-1}\right)\left(M V M^{-1}\right) \leqq M U V M^{-1}$. 
Each connector system $\mathfrak{I}$ on $R$ can be used to construct a connector system on $S$ by a transfer of each $U \in \mathfrak{D}$ to the connector $M U M^{-1}$ on $S$. $M \mathfrak{S} M^{-1}$ denotes the connector system $\left\{M U M^{-1}: U \in \mathfrak{D}\right\}$. The following propositions are proved by referring to $(6.1),(6.2),(6.3)$ and (6.4).

(6.5) $M \mathfrak{I}^{<} M^{-1} \subseteq\left(M \mathfrak{D} M^{-1}\right)^{<}$and $\left(M \mathfrak{D}^{<} M^{-1}\right)^{<}=\left(M \mathfrak{D} M^{-1}\right)^{<}$.

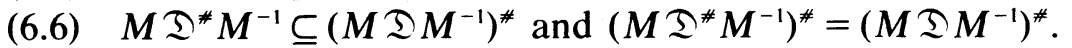

(6.7) $M \mathfrak{D}^{\times} M^{-1}=\left(M \mathfrak{D} M^{-1}\right)^{\times}$.

(6.8) If $\mathfrak{I}$ is a net, then $M \mathfrak{D} M^{-1}$ is a net.

(6.9) If $\mathfrak{I}$ is a prenet, then $M \mathfrak{I} M^{-1}$ is a prenet.

Proof. Refer to (6.6), (1.23) and (6.8).

(6.10) If $\mathfrak{D}$ is topological then $M \mathfrak{D} M^{-1}$ is topological.

(6.11) If $\mathfrak{I}$ is uniform then $M \mathfrak{D} M^{-1}$ is uniform.

(6.12) If $\mathfrak{I}$ is a pretopology then $M \mathfrak{I} M^{-1}$ is a pretopology.

Proof. $\left(M \Omega^{*} M^{-1}\right)^{*}$ is a topological net by $(6.8),(6.10),(1.23)$ and (3.1), if $I$ is a pretopology. Hence, by $(6.6),\left(M \cap M^{-1}\right)^{*}$ is a topological net.

(6.13) $I$ and 5 are connector systems on $R . M I M^{-1}$ is stronger than $M\left(5 M^{-1}\right.$ if $\mathfrak{D}$ is stronger than $\mathcal{E}$.

(6.14) $M I M^{-1}$ is finer than $M \mathfrak{F} M^{-1}$ if $\mathbb{I}$ is finer than $\mathfrak{E}$.

$Y M^{-1}$ denotes the inverse image of a set $Y \subseteq R$ by a mapping $M$.

(6.15) $\mathfrak{I}$ is a prenet on $R$ and $\mathscr{T}(\mathfrak{I})$ is the open-topology induced by $\Sigma$. $\mathscr{T}\left(M \cap M^{-1}\right)$ is the open-topology on $S$ induced by $M \cap M^{-1}$. Then, $\left\{Y M^{-1}: Y \in \mathscr{T}(I)\right\} \subseteq \mathscr{T}\left(M \cong M^{-1}\right)$.

(6.16) $\left\{Y M^{-1}: Y \in \mathscr{T}(D)\right\}=\mathscr{T}\left(M \cong M^{-1}\right)$ if $\mathfrak{I}$ is a pretopology.

Proof. If $X \in \mathscr{T}\left(M I M^{-1}\right)$ and $x \in X$, then there is $U(x) \in \mathbb{I}$ such that $x\left(M U(x) M^{-1}\right) \subseteq X$. According to the interior theorem, since $\mathfrak{I}$ is a pretopology, $y=x M$ belongs to $I-\operatorname{Int}(y U(x)) \in \mathscr{T}(I)$, and I-Int $(y U(x)) \subseteq y U(x)$. Let $Y=U\{$ I-Int $(y U(x)): y=x M$, $x \in X\}$. Then $Y \in \mathscr{T}(\Sigma)$ and $X \subseteq Y M^{-1} \subseteq \cup\left\{(y U(x)) M^{-1}: y=\right.$ $x M, x \in X\} \subseteq X$. Hence, $X=Y M^{-1}$.

$\mathfrak{P}$ is a connector system on $S$ and $I$ is a connector system on $R$. A mapping $M$ from $S$ to $R$ is called $\mathfrak{Y}$-continuous w.r.t. $I$ if $\mathfrak{Y}$ is finer than $M \cong M^{-1}$. This is a generalization of continuity. Neither connector system needs to be a topology.

(6.17) $M$ is $\mathfrak{P}$-continuous w.r.t. $I$ if and only if for each $U \in I$ and for each $x \in S$, there are $V_{i} \in \mathfrak{N}, i=1,2,3, \cdots, n$ such that $\cap\left\{x V_{i}: i=1,2,3, \cdots, n\right\} \subseteq x\left(M U M^{-1}\right)$.

(6.18) $\mathfrak{A}$ is a prenet. $\quad M$ is $\mathfrak{A}$-continuous w.r.t. $\mathfrak{I}$ if and only if for each $U \in \mathfrak{D}$ and for each $x \in S$, there is $V \in \mathfrak{A}$ such that $(x V) M \subseteq$ $(x U) M$. 
Continuity Theorem. $\mathfrak{A}$ and $\mathfrak{D}$ are connector systems on $S$ and $\boldsymbol{R}$ respectively. $\mathfrak{A}$ is a prenet and $\mathfrak{D}$ is a pretopology. $\quad M$ is a mapping from $S$ to $R . \quad M$ is $\mathfrak{A}$-continuous w.r.t. $\mathfrak{D}$ if and only if $\left\{Y M^{-1}: Y \in\right.$ $\mathscr{T}(\mathfrak{D})\} \subseteq \mathscr{T}(\mathfrak{A})$.

Proof. $M \mathfrak{D} M^{-1}$ is a pretopology if $\mathfrak{D}$ is a pretopology. (4.1) and (4.5) $\Rightarrow$ a prenet $\mathfrak{A}$ is finer than a pretopology $M \mathfrak{D} M^{-1}$ if and only if $\mathscr{T}\left(M \mathfrak{D} M^{-1}\right) \subseteq \mathscr{T}(\mathfrak{U})$. Hence, by (6.16), $\mathfrak{A}$ is finer than $M \mathfrak{D} M^{-1}$ if and only if $\left\{Y M^{-1}: Y \in \mathscr{T}(\mathfrak{D})\right\} \subseteq \mathscr{T}(\mathfrak{A})$.

Comment on the continuity theorem: An implication of the theorem is a compatibility of continuous mappings and continuous functions from a topological space $(S, \mathscr{T}(\mathfrak{U}))$ to a topological space $(R, \mathscr{T}(\mathfrak{D}))$. The theorem states that if $\mathfrak{A}$ is a prenet and $\mathfrak{I}$ is a pretopology, then a continuous mapping is topologically continuous and vice versa.

$\mathfrak{A}$ is a connector system on $S$ and $\mathfrak{D}$ is a connector system on $R$. A mapping $M$ from $S$ to $R$ is called uniformly $\mathfrak{Q}$-continuous w.r.t. $\mathfrak{D}$ if $\mathfrak{A}$ is stronger than $M \mathfrak{D} M^{-1} . \mathfrak{A}$ is stronger, then $\mathfrak{A}$ is finer, therefore, $M$ is $\mathfrak{A}$-continuous if $M$ is uniformly $\mathfrak{A}$-continuous. implies the converse if $\mathfrak{U}$ is a sharp prenet.

(6.19) $M$ is uniformly $\mathfrak{A}$-continuous w.r.t. $\mathfrak{D}$ if and only if for each $U \in \mathfrak{D}$, there are $V_{i} \in \mathfrak{A}, i=1,2,3, \cdots, n$, such that $\cap V_{i} \leqq$ $M U M^{-1}$.

(6.20) $\mathfrak{A}$ is a sharp prenet. $\quad M$ is $\mathfrak{A}$-continuous w.r.t. $\mathfrak{I}$ if and only if $M$ is uniformly $\mathfrak{A}$-continuous w.r.t. $\mathfrak{D}$.

Kernel Theorem. $\mathfrak{T}$ is a topology on $S, \mathfrak{U}$ is the uniformity kernel of $\mathfrak{T}$ and $\mathfrak{D}$ is a uniform net on $R$. If a mapping $M$ from $S$ to $R$ is $\mathfrak{I}$-continuous w.r.t. $\mathfrak{D}$, then $M$ is uniformly $\mathfrak{U}$-continuous w.r.t. $\mathfrak{I}$.

Proof. The continunity of $M$ implies $M \mathfrak{D} M^{-1} \subseteq \mathfrak{T} . \quad M \mathfrak{D} M^{-1}$ is a uniform net by (6.8) and (6.11), if $\mathfrak{D}$ is a uniform net. Then, $\left(M \mathfrak{D} M^{-1}\right)^{<}$ is the uniformity hull of $M \mathfrak{D} M^{-1}$, and $M \mathfrak{D} M^{-1} \subseteq\left(M \mathfrak{D} M^{-1}\right)^{<} \subseteq \mathfrak{H} \subseteq$ $\mathfrak{I}$. Hence, $M$ is uniformly $\mathfrak{H}$-continuous w.r.t. $\mathfrak{D}$.

Comment on the kernel theorem: A uniform net is a pretopology by (3.12) and (5.1), and a topology is a prenet. Therefore, by the continuity theorem, the kernel theorem can be applied to a continuous function from a topological space $(S, \mathscr{T}(\mathfrak{I}))$ to a topological space $(R, \mathscr{T}(\mathfrak{D}))$. (5.6) states that $\mathfrak{D}^{<}$is the uniformity hull of a uniform net D. Since $\mathscr{T}(\mathfrak{D})=\mathscr{T}\left(\mathfrak{D}^{<}\right), \quad \mathscr{T}(\mathfrak{D})$ is a topology induced by a uniformity. The kernel theorem presents an answer to the following question in a generalized form. If $S$ is a topological space and $R$ is a uniform-topological space, then what is a uniformity on $S$, for which every continuous function from $S$ to $R$ is uniformly continuous? 
7. Mappings. Let $R_{\lambda}, \lambda \in \Lambda$ be a system of spaces and let $\mathfrak{I}_{\lambda}$ be a connector system on $R_{\lambda}$ for each $\lambda \in \Lambda$. If a topology hull $\mathfrak{I}$ of $\cup\left\{M_{\lambda} \mathfrak{D}_{\lambda} M_{\lambda}^{-1}: \lambda \in \Lambda\right\}$ exists, then $\mathfrak{I}$ is called the weak topology on $S$ by $\left\{M_{\lambda}: \lambda \in \Lambda\right\}$, w.r.t. $\left\{\mathfrak{D}_{\lambda}: \lambda \in \Lambda\right\}$. $\mathfrak{I}$ is the weakest topology among those for which $M_{\lambda}, \lambda \in \Lambda$ are continuous.

(7.1) $\mathfrak{T}$ is the weak topology by $\left\{M_{\lambda}: \lambda \in \Lambda\right\}$, w.r.t. $\left\{\mathfrak{D}_{\lambda}: \lambda \in\right.$ $\Lambda$ \}. If $\mathfrak{D}_{\lambda} \subseteq \mathfrak{F}_{\lambda} \subseteq \mathfrak{D}_{\lambda}^{\times *<}$ for every $\lambda \in \Lambda$, then $\mathfrak{T}$ is the weak topology by those mappings w.r.t. $\left\{\mathscr{F}_{\lambda}: \lambda \in \Lambda\right\}$.

Proof. Refer to (1.5), (1.9), (1.22), (6.5), (6.6) and (6.7).

(7.2) If $\mathfrak{D}_{\lambda}, \lambda \in \Lambda$ are all topological or all of them are pretopologies, then there exists a weak topology by every system of mappings, w.r.t. those connector systems.

Proof. Refer to (6.10), (6.12), (3.3) and (3.13).

Weak Topology Theorem. $\mathfrak{I}$ is a topology on $S$. $\mathfrak{I}_{\lambda}$ is a connector system on $R_{\lambda}$ and $M_{\lambda}$ is a $\mathfrak{I}$-continuous mapping, w.r.t. $\mathfrak{D}_{\lambda}$ from $S$ to $R_{\lambda}$, for each $\lambda \in \Lambda$. $\mathfrak{I}$ is the weak topology on $S$ by those mappings, w.r.t. the given connector systems if for each $U \in \mathfrak{T}$ and for each $x \in S$, there is $\lambda \in \Lambda$ and $V \in \mathfrak{D}_{\lambda}$ such that $x\left(M_{\lambda} V M_{\lambda}^{-1}\right) \subseteq x U$.

Proof. The hypotheses are: $M_{\lambda} \mathfrak{D}_{\lambda} M_{\lambda}^{-1} \subseteq \mathfrak{T}$ for every $\lambda \in \Lambda$ and

$$
\begin{gathered}
\mathfrak{I} \subseteq\left(\cup M_{\lambda} \mathfrak{D}_{\lambda} M_{\lambda}^{-1}\right)^{\#<} . \\
\left(\cup M_{\lambda} \mathfrak{I}_{\lambda} M_{\lambda}^{-1}\right)^{\#<} \subseteq\left(\cup M_{\lambda} \mathfrak{I}_{\lambda} M_{\lambda}^{-1}\right)^{\times *} \subseteq \mathfrak{I}^{\times *<}=\mathfrak{I} .
\end{gathered}
$$

Hence, $\mathfrak{I}$ is the topology hull of $\cup M_{\lambda} \mathfrak{I}_{\lambda} M_{\lambda}^{-1}$.

If there exists a uniformity hull of $\cup M_{\lambda} \mathfrak{D}_{\lambda} M_{\lambda}^{-1}$, then the uniformity hull is called the weak uniformity by those mappings, w.r.t. the given connector systems. A weak uniformity is the weakest uniformity among those for which each $M_{\lambda}$ is uniformly continuous w.r.t. $\mathfrak{I}_{\lambda}$.

(7.3) $\mathbb{U}$ is a weak uniformity w.r.t. $\left\{\Sigma_{\lambda}: \lambda \in \Lambda\right\}$. If $\Sigma_{\lambda} \subseteq \mathfrak{F}_{\lambda} \subseteq$ $\Omega_{\lambda}^{x<}$ for each $\lambda \in \Lambda$, then $\mathbb{U}$ is the weak uniformity by the same mappings, w.r.t. $\left\{\mathscr{E}_{\lambda}: \lambda \in \Lambda\right\}$.

WEAK UNIFORMITY THEOREM. $\mathfrak{D}_{\lambda}$ is a unifrom connector system on $R_{\lambda}$ and $M \lambda$ is a mapping from $S$ to $R_{\lambda}$ for each $\lambda \in \Lambda$. Then there exists a weak uniformity $\mathfrak{U}$ by $\left\{M_{\lambda}: \lambda \in \Lambda\right\}$, w.r.t. $\left\{\mathfrak{D}_{\lambda}: \lambda \in \Lambda\right\}$, and the topology hull of $\mathfrak{U}$ is the weak topology by those mappings, w.r.t. the given connector systems. 
Proof. Let $\mathfrak{B}=\cup M_{\lambda} \mathfrak{D}_{\lambda} M_{\lambda}^{-1}$ and let $\mathfrak{U}=\mathfrak{B}^{\times<} . \quad \mathfrak{B}$ is uniform and $\mathfrak{U}$ is the uniformity hull by (6.11), (5.3) and (5.4). $\quad \mathfrak{U}^{\times *<}$ is the topology hull of $\mathfrak{B}$ by (3.3) and (3.4), as well as that of $\mathfrak{U}$. Hence the topology hull of $\mathbb{U}$ is the weak topology by $\left\{M_{\lambda}: \lambda \in \Lambda\right\}$.

Let $M_{\lambda}, \lambda \in \Lambda$ be mapping from $S$ to $R$ and let $U$ be a connector on $R$. Let $\cap M_{\lambda} U M_{\lambda}^{-1}$ denote the connector, $x \in S \rightarrow \cap\left\{(y U) M_{\lambda}^{-1}: y=\right.$ $x M_{\lambda}, \lambda \in \Lambda$.

(7.4) $U$ and $V$ are connectors on $R$ and $W$ is the product of $\cap M_{\lambda} U M_{\lambda}^{-1}$ and $\cap M_{\lambda} V M_{\lambda}^{-1}$. Then $W \leqq \cap M_{\lambda} U V M_{\lambda}^{-1}$.

(7.5) $\left\{\cap M_{\lambda} U M_{\lambda}^{-1}: U \in \mathbb{I}^{<}\right\} \subseteq\left\{\cap M_{\lambda} U M_{\lambda}^{-1}: U \in \mathbb{I}\right\}^{<}$.

(7.6) $\left\{\cap M_{\lambda} U M_{\lambda}^{-1}: U \in \mathfrak{I}^{\times}\right\}=\left\{\cap M_{\lambda} U M_{\lambda}^{-1}: U \in \mathfrak{I}\right\}^{\times}$.

(7.7) If $\mathfrak{I}$ is topological then $\left\{\cap M_{\lambda} U M_{\lambda}^{-1}: U \in \mathfrak{I}\right\}$ is topological.

(7.8) If $\mathfrak{I}$ is uniform then $\left\{\cap M_{\lambda} U M_{\lambda}^{-1}: U \in \mathfrak{I}\right\}$ is uniform.

$\mathfrak{I}$ is a connector system on $S$ and $\mathfrak{I}$ is a connector system on $R$. A system of mappings $M_{\lambda}, \lambda \in \Lambda$ from $S$ to $R$ is called $\{$-equi-continuous w.r.t. $\mathfrak{I}$ if $\mathfrak{P}$ is finer than $\left\{\cap M_{\lambda} U M_{\lambda}^{-1}: U \in \mathfrak{I}\right\}$, i.e., the latter is included in $\mathfrak{P}^{\times \#<}$. The mappings are called uniformly $\mathfrak{P}$-equi-continuous if $\mathfrak{H}$ is stronger than $\left\{\cap M_{\lambda} U M_{\lambda}^{-1}: U \in \mathfrak{D}\right\}$, i.e., the latter is included in $\mathfrak{P}^{\times<}$. The uniformly equi-continuity implies uniform continuity of each mapping in the system. If there exists a topology hull of $\{\cap$ $\left.M_{\lambda} U M_{\lambda}^{-1}: U \in I\right\}$ then the topology hull is called the equi-topology by $\left\{M_{\lambda}: \lambda \in \Lambda\right\}$.

(7.9) $I$ and $\mathbb{F}$ are connector systems on $R$ and $I \subseteq E \subseteq I^{x<}$. If $\mathfrak{I}$ is an equi-topology by a system of mappings to $R$, w.r.t. $\mathfrak{I}$ then $\mathfrak{I}$ is the equi-topology by the same mappings, w.r.t. 5 .

(7.10) $\left\{\cap M_{\lambda} U M_{\lambda}^{-1}: U \in I\right\}^{\star \#<}$ is the equi-topology by $\left\{M_{\lambda}: \lambda \in\right.$ $\Lambda$ \}, w.r.t. $I$ if $I$ is topological.

Proof. Refer to (7.7) and (3.3).

If there exists a uniformity hull of $\left\{\cap M_{\lambda} U M_{\lambda}^{-1}: U \in \Sigma\right\}$ then the uniformity hull is called the equi-uniformity by $\left\{M_{\lambda}: \lambda \in \Lambda\right\}$, w.r.t. $₹$, i.e., it is the weakest uniformity for which the mappings are uniformly equi-continuous.

(7.11) $I$ and $\mathbb{E}$ are connector systems on $R$ and $I \subseteq[\subseteq$ $I^{x<}$. If $\mathbb{l}$ is an equi-uniformity by a system of mappings w.r.t. $I$, then $\mathbb{I I}$ is the equi-uniformity by the same mappings w.r.t. .

(7.12) If $I$ is uniform then $\left\{\cap M_{\lambda} U M_{\lambda}^{-1}: U \in \mathfrak{I}\right\}^{\times<}$is the equiuniformity by $\left\{M_{\lambda}: \lambda \in \Lambda\right\}$, w.r.t. $I$ and the equi-topology is the topology hull of the equi-uniformity.

Proof. Refer to (7.8), (5.4), (3.5) and (7.10). 
8. Uniformalizable topologies. The set of all real numbers is denoted by $(-\infty, \infty)$. For each positive $\epsilon$, let $U(\epsilon)$ denote the connector on $(-\infty, \infty)$ such that $x U(\epsilon)=\{y:|x-y| \leq \epsilon\}$ for every $x \in(-\infty, \infty)$. Then the connector system $\{U(\epsilon): 0<\epsilon\}$ is a uniform net. This is the only connector system, in this paper, we deal with for the real numbers. $\mathfrak{U}[a, b]$ denotes the connector system $\mathfrak{I}(-\infty, \infty)$ if each connector is restricted on a closed interval [a.b].

$f$ is a function from a space $S$ to $(-\infty, \infty)$. We write $a \leqq f \leqq b$ if the image of $f$ is bounded by $a$ and $b$. A topology $\mathfrak{I}$ on $S$ is called completely regular if for each $U \in \mathfrak{I}$ and for each $x \in S$, there is a $\mathfrak{I}$-continuous function $f$ from $S$ to $[0,1]$, w.r.t. $\mathfrak{M}[0,1]$ such that $x f=1$ and $y f=0$ if $y$ does not belong to $x U$.

The above definition is obviously compatible with the definition of a completely regular topology on the topological space $(S, \mathscr{T}(\mathfrak{I}))$.

(8.1) If $\mathfrak{I}$ is completely regular then $\mathfrak{I}$ is a weak topology by a system of functions.

Proof. Refer to the weak topology theorem in $\$ 7$.

(8.2) A weak topology by functions, w.r.t. $\mathfrak{H}(-\infty, \infty)$ is completely regular.

Uniformalization Theorem. A topology is the topology hull of a uniformity if and only if it is completely regular.

Proof. If $\mathfrak{I}$ is completely regular then $\mathfrak{I}$ is a weak topology and by the weak uniformity theorem in $\S 7$, $\mathfrak{I}$ is the topology hull of the weak uniformity by the same functions which induce the weak topology. Conversely, if $\mathfrak{I}$ is the topology hull of a uniformity $\mathbb{l}$ then, since $\mathfrak{I}=\mathbb{1}^{*}$, for each $U \in \mathcal{I}$ and for each $x \in S$, there is $V \in \mathbb{l}$ such that $x U=x V$. Theorem 5 in $\$ 31$ of [1] and Theorem 19.1 of [2] state that there is a uniformly continuous function $f$ such that $0 \leqq f \leqq 1$, $x f=1$ and $y f=0$ if $y$ does not belong to $x V$.

9. Bounded connectors. A connector $U$ on a space $S$ is called bounded if there are $x_{i} \in S, i=1,2,3, \cdots, n$ such that $S=$ $\cup\left\{x_{i} U: i=1,2,3, \cdots, n\right\}$. A connector $U$ is called absolutely bounded if for each nonempty set $X \subseteq S$, there are $x_{i} \in X, i=1,2,3, \cdots, n$ such that $X \subseteq \cup\left\{x_{i} U: i=1,2,3, \cdots, n\right\}$. If $U \leqq V$ and $U$ is bounded or absolutely bounded, then $V$ is bounded or absolutely bounded respectively.

A connector system $\because$ on $S$ is called totally bounded if every connector in $\mathfrak{P}$ is bounded. This is a generalization of totally bounded uniformities. 
(9.1) If a connector system $\mathfrak{Y}$ is uniform and totally bounded then every connector in $\mathfrak{P}$ is absolutely bounded.

Proof. For each $U \in \mathfrak{Y}$, since $\mathfrak{P}$ is uniform, there is $V \in \mathfrak{Y}$ such that $V^{-1} V \leqq U$. Let $X$ be a nonempty subset of $S$. There are $x_{1} \in S$, $i=1,2,3, \cdots, n$ such that $X$ is included in the union of $x_{i} V, i=$ $1,2,3, \cdots, n$ and $X \cap x_{i} V$ is nonempty for every $i=1,2,3, \cdots, n$. If $y_{i} \in X \cap x_{i} V, i=1,2,3, \cdots, n$, then, $x_{l} V \subseteq y_{i} V^{-1} V \subseteq y_{i} U$ thus $X$ is included in the union of $y_{i} U, i=1,2,3, \cdots, n$ and each $y_{i}$ belongs to $X$. Hence, $\mathbb{l l}$ is absolutely bounded.

A topology $\mathfrak{I}$ on $S$ is called compact if the open-topology $\mathscr{T}(\mathfrak{I})$ is compact (in usual sense), i.e., every open covering of $S$ has a finite subcovering.

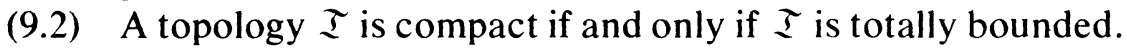

Proof. The basis theorem in $\S 4$ states that there is an open connector $V \in \mathcal{Y}$ for each $U \in \mathcal{Y}$, such that $V \leqq U$. If $\mathcal{I}$ is compact then, since all $x V$ are $₹$-open, $V$ is bounded. Hence, $I$ is totally bounded if $\Im$ is compact. Conversly, suppose $S=\cup\left\{X_{\lambda}: \lambda \in \Lambda\right\}$ for some $X_{\lambda} \in \mathscr{T}(I), \lambda \in \Lambda$. Correspond each $x \in S$ to one of these $X_{\lambda}$ which contains $X_{\lambda}$ which contains $x$ and define a connector $U$. Then $U$

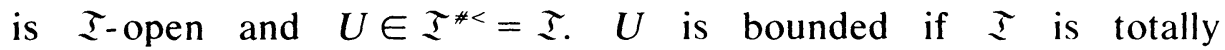
bounded. Therefore, there are $x_{i} \in S, i=1,2,3, \cdots, n$ such that $S$ is the union of $x_{i} U, i=1,2,3, \cdots, n$, which is a finite union of some $X_{\lambda}$. $\lambda \in \Lambda$.

Comment on (9.2). (9.2) is a generalization of a theorem on uniform spaces. The open-topology $\mathscr{T}(\mathfrak{I})$ in $(9.2)$ is not necessarily a uniform topology. In fact, the theorem is a characterization of the compact topologies because each open-topology is induced by a topology.

Compact Topology Theorem. If the topology hull $11^{*}$ of a uniformity $\|$ is compact then $\|$ is the uniformity kernel of $\|^{*}$.

Proof. First, we prove that a real valued 11 -continuous function on $S$ is uniformly continuous if $\mathbb{1}^{*}$ is compact. By the definition of continuity, for each positive $\epsilon$ and $x \in S$, there is $U \in \| l$ such that $|x f-y f|<\epsilon / 2$ if $y \in x U$. There is $V \in U$ such that $V^{2} \leqq U$, and $|x f-y f|<\epsilon / 2$ if $y \in x V^{2}$. By corresponding $x V$ to $x$, we obtain a connector $W \in \mathbb{l}^{*}$. Since $\mathbb{l}^{*}$ is totally bounded by. (9.2), there are $x_{i} \in S, i=1,2,3, \cdots, n$ such that $S$ is included in the union of $x_{i} W$, $i=1,2,3, \cdots, n$. By the definition of $W$, there are corresponding $V, \in$ Ul, $i=1,2,3, \cdots, n$ such that $x_{\imath} W=x_{i} V_{\iota}$, and $\left|x_{\imath} f-y f\right|<\epsilon / 2$ if 
$y \in x_{i} V_{i}^{2}$. Let $\quad V=\cap V_{i}$. Then $\quad|x f-y f|<\epsilon \quad$ whenever $y \in x V$. Hence, $f$ is uniformly ll-continuous. Let $\mathfrak{R}$ be the uniformity kernel of $\mathfrak{l l}^{*}$. $\mathbb{1 1}^{*}$ is totally bounded and $\mathfrak{B} \subseteq \mathfrak{l}^{*}$, thus $\mathfrak{R}$ is totally bounded. Every uniformly $\mathfrak{B}$-continuous function is $\mathbb{1 1}$-continuous, thus it is uniformly $\mathbb{l l}$-continuous. Therefore, $\mathfrak{Q} \subseteq \mathbb{l}$ by Theorem 6 in $\S 33$ of [1]. $\quad \mathbb{\subseteq} \subseteq \mathfrak{B}$ always holds because $\mathfrak{P}$ is the strongest uniformity included in $11^{*}$. Hence, $\mathbb{l l}$ is the uniformity kernel of $1^{*}$.

10. Semi-bounded connectors. A connector $U$ on a space $S$ is called semi-bounded if there is a positive integer $n$ such that $U^{n}$ is bounded. $U^{n}$ is defined by induction, i.e., $U^{n}=U^{n-1} U$. A connector $U$ is called absolutely semi-bounded if for each nonempty set $X \subseteq S$, there are $x_{i} \in X, i=1,2,3, \cdots, m$ and a positive integer $n$ such that $X \subseteq \cup\left\{x_{i} U^{n}: i=1,2,3, \cdots, m\right\}$. If $U \leqq V$ and $U$ is semi-bounded or absolutely semi-bounded, then $V$ is semi-bounded or absolutely semi-bounded respectively.

A connector system $\mathfrak{P}$ is called bounded if every connector in ?I is semi-bounded.

(10.1) A uniformity $\mathbb{l l}$ is bounded if and only if every uniformly II-continuous function is bounded.

Proof. Refer to Theorem 2 in $\$ 32$ of [1].

A connector system $: T$ is called absolutely bounded if every connector in $: \mathbb{I}$ is absolutely semi-bounded.

(10.2) A uniformity $\mathbb{l}$ is absolutely bounded if and only if $\mathbb{l l}$ is totally bounded.

Proof. Refer to Theorem 2 in $\$ 33$ of [1].

A topology $\mathfrak{I}$ on a space $S$ is called pseudo-compact if every $\boldsymbol{\Gamma}$-continuous function (real-valued) is bounded.

Pseudo-Compact Topology Theorem. A topology $\mathfrak{I}$ is pseudocompact if and only if the uniformity kernel $l l$ of $\xi$ is bounded.

Proof. If $l l$ is bounded then, since every $I$-continuous function is uniformly $\mathbb{I l}$-continuous by the kernel theorem in $\$ 6$, $₹$ is pseudocompact. On the other hand, every uniformly 11 -continuous function

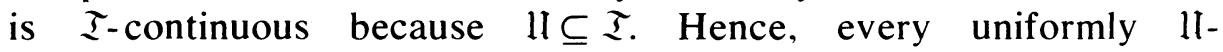
continuous function is bounded if $\mathcal{I}$ is pseudo-compact. By (10.1), 11 is bounded. 


\section{REFERENCES}

1. H. Nakano, Topology and linear topological spaces, Tokyo, 1951.

2. - Uniform spaces and transformation groups, Wayne State University Press, 1968.

Received August 1, 1973.

Wayne State University

AND

State University of New York. College at Brockport 




\section{Pacific Journal of Mathematics}

\section{Vol. 56, No. $1 \quad$ November, 1975}

Shimshon A. Amitsur, Central embeddings in semi-simple rings .......... 1

David Marion Arnold and Charles Estep Murley, Abelian groups, A, such

that $\operatorname{Hom}(A,---)$ preserves direct sums of copies of $A \ldots \ldots \ldots .$.

Martin Bartelt, An integral representation for strictly continuous linear

operators ................................... 21

Richard G. Burton, Fractional elements in multiplicative lattices......... 35

James Alan Cochran, Growth estimates for the singular values of

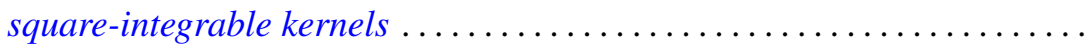

C. Martin Edwards and Peter John Stacey, On group algebras of central

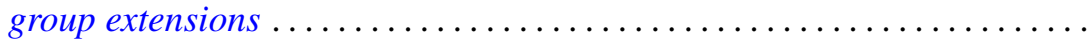

Peter Fletcher and Pei Liu, Topologies compatible with homeomorphism

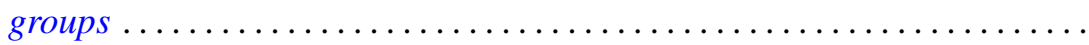

George Gasper, Jr., Products of terminating ${ }_{3} F_{2}(1)$ series ............ 87

Leon Gerber, The orthocentric simplex as an extreme simplex ............

Burrell Washington Helton, A product integral solution of a Riccati

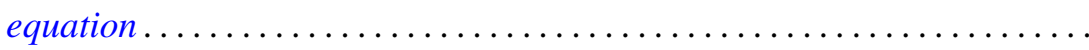

Melvyn W. Jeter, On the extremal elements of the convex cone of

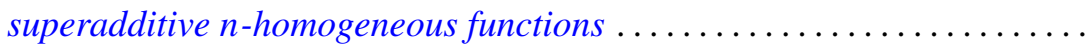

R. H. Johnson, Simple separable graphs .

Margaret Humm Kleinfeld, More on a generalization of commutative and

alternative rings. . .

A. Y. W. Lau, The boundary of a semilattice on an $n$-cell.

Robert F. Lax, The local rigidity of the moduli scheme for curves ...

Glenn Richard Luecke, A note on quasidiagonal and quasitriangular

operators .

Paul Milnes, On the extension of continuous and almost periodic functions

Hidegoro Nakano and Kazumi Nakano, Connector theory.

James Michael Osterburg, Completely outer Galois theory of perfect rings ..................................

Lavon Barry Page, Compact Hankel operators and the F. and M. Riesz

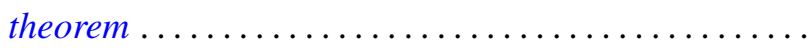

Joseph E. Quinn, Intermediate Riesz spaces..................... 225

Shlomo Vinner, Model-completeness in a first order language with a generalized quantifier.

Jorge Viola-Prioli, On absolutely torsion-free rings ..........

Philip William Walker, A note on differential equations with all solutions of integrable-square............................

Stephen Jeffrey Willson, Equivariant maps between representation 